\title{
Experience of a Tertiary-Level Urology Center in the Clinical Urological Events of Rare and Very Rare Incidence. I. Surgical Never Events: 1. Urological Wrong-Surgery Catastrophes and Disabling Complications
}

\author{
Rabea A. Gadelkareem \\ Assiut Urology and Nephrology Hospital, Faculty of Medicine, Assiut University, Assiut, Egypt
}

\section{Key Words}

Never events • Surgical catastrophes •

Urological complications

\begin{abstract}
Introduction: Surgical never events are preventable harmful non-intentional human errors. Urology is a common surgical specialty for their occurrence. Patients and Methods: A retrospective search of our center's data was done during the period 2006-2016 for surgical never events. Each included case was studied for the primary diagnosis, procedure, and subspecialty, never event type and timing, needed extra-interventions, urologist/procedure proportioning, outcomes, and possible underlying causes of the event. Results: Of more than 55,000 different urological interventions, 61 patients were involved in never events. Wrong procedures represented $75 \%$ of the never events, and endourology and urolithiasis subspecialties were more often involved. The main detectable underlying factor was the disproportion between the levels of the procedure class and the qualification of the urologist (41\%). Thirty-four cases had extra-procedures. The short-term harm effect represented the final outcome in $42 \%$ of all events. Death, permanent organ loss, and long-term harm represented 20,15 , and $23 \%$, respectively. Conclusion: Urological surgical never events are rare, but their final outcomes could be catastrophic, even leading to death.

Copyright $\odot 2017$ S. Karger AG, Basel
\end{abstract}

\section{Introduction}

The term "sentinel event" refers to a wide spectrum of adverse events of unexpected and unfavorable occurrence that results from error or unknown cause leading to defects or risks in human healthcare. It was defined by the Joint Commission as a "patient safety event (not primarily related to the natural course of the patient's illness or underlying condition) that reaches a patient and results in any of the following: death, permanent harm, or severe temporary harm" [1]. This spectrum includes serious surgical complications and unfavorable outcomes. In urology, they are associated with or follow deviations of the clinical course of surgeries involving or affecting the genitourinary organs [2]. Urological complications are classified as iatrogenic when they result from non-intentional error in the surgical practice by trained and licensed surgeons or persons for surgical intervention. However, they are criminal when they result from inadequately trained or unlicensed personnel [3]. Moreover, serious surgical complications and events which are due to non-intentional mistakes of clinicians and medical personnel may contribute to catastrophic or disastrous outcomes that should never occur as sequels or complications [4]. These outcomes are termed "surgical never events" and their final outcome may be death, permanent loss of an organ or its function, long-standing disability, mutilation, or serious psychological impacts $[5,6]$. They 


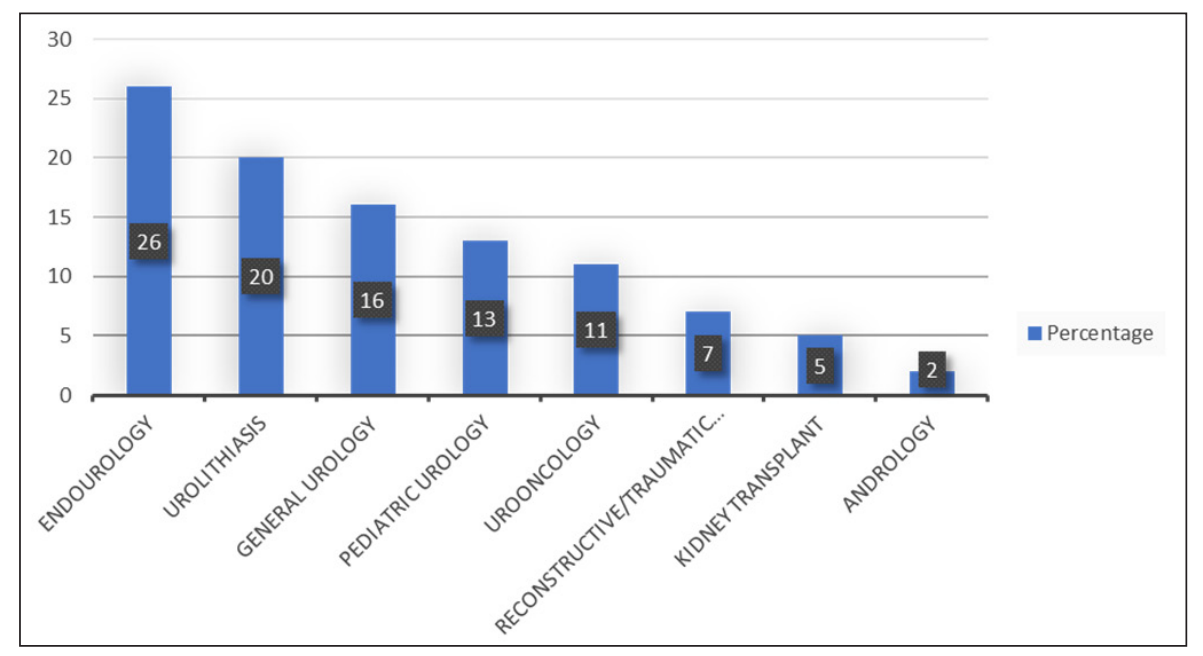

Fig. 1. Percentage distribution of the wrong-surgery never events per urological subspecialty.

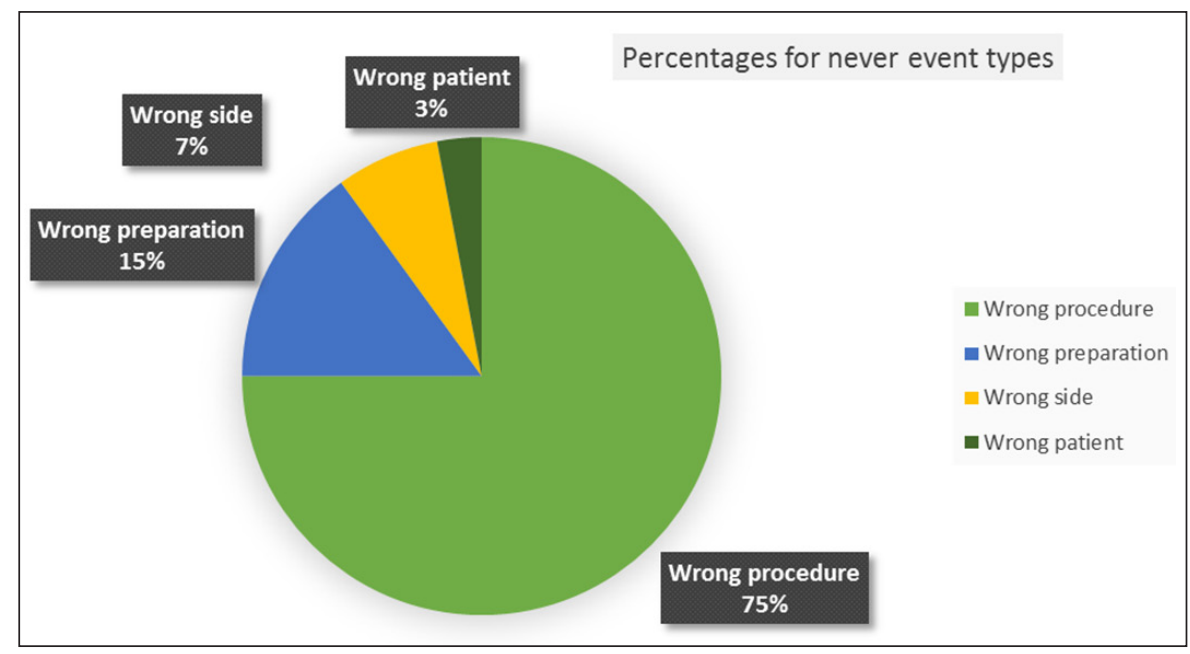

Fig. 2. Type distribution of the wrong-surgery never events.

were included in the serious healthcare reportable surgical events by the National Quality Forum and the Joint Commission in United States of America [6].

Although surgical never events seem rare [6], their worldwide current incidence is poorly understood [7, 8]. In Egypt, study of the urological surgical never events is nonexistent in scientific urological publications. Shame and reluctance from exposing these events could be the underlying factor [9]. In the current study, however, the target was the never events which occurred in our terti- ary-level urology center. This study may help establish the first step for a preventive plan by defining the magnitude and features of the event.

\section{Patients and Methods}

A retrospective revision of the operative, endourological, and outpatient surgical interventions in the Assiut Urology and Nephrology Hospital, Assiut University was done for surgical never events in the time period of July 2006 - June 2016. The never 
events which were included in this study fulfilled the definition and criteria reported by National Quality Forum Update of 2011: to be adverse, serious, unambiguous, measurable, largely preventable, indicative of a problem in the healthcare system, and important for public credibility [6]. Cases which were done outside our hospital, but presented to us for management of the never event, were also included. A serious event means an event leading to death (American Society of Anesthesiologists class I patient), disability, or organ loss without being described as an acceptable event in the primary target surgery. Each case was studied for age, gender, surgical never event type, involved organ, primary diagnosis and urological subspecialty, primary procedure (classified as minor, intermediate, or major), operator qualification, operator-procedure levels proportion, possible explanation (mere mistake or presence of a predisposing underlying factor), number of salvage extra-procedures, final outcomes, and medico-legal disputes. The time of occurrence of the events was classified into 2 categories (before and after 2010) in relation of the clear demarcation between all the urological subspecialties in our hospital and corrective administrative actions. Moreover, many administrative steps were carried out towards the surgeons and methods of maintenance of the operative armamentarium. Final outcomes were classified into death (when it occurred intraoperative or directly postoperative up to 48 hours or as a surgical sequel of an organ function loss that did not resolve or was corrected), organ or complete organ function loss, long-term or permanent harm (other than the previous items), and short-term or temporary serious harm (events that threaten the patient's life or organ function, but were resolved, corrected, or completely treated). Complications or deaths which are known as natural sequels or complications of the primary disease, or high-risk procedures, such as pulmonary embolism after major and pelvic surgeries, anesthesia-related events, and surgical site infections were not included in the study. Also, due to differences in the nature of retained or missed urological surgical items, they were excluded from this article for study in a separate article.

\section{Results}

According to our annual rate of the urological interventions between July 2006 and June 2016, there were more than 55,000 interventions that varied from the insertion of a percutaneous nephrostomy tube to kidney transplantation. Of them, 61 urological surgical never events were detected representing $0.11 \%$ or about 6 events per year. Thirty-seven events $(61 \%)$ occurred before the year 2010, while only 24 events (39\%) occurred after. There were 46 male and 15 female patients. The age range was 3-76 years and only 8 children were included. Twelve of the 61 cases $(20 \%)$ were operated on outside of our hospital, but were referred to our hospital for management.

The distribution of the never events according to the urological subspecialty, under which the primary procedure was planned, is illustrated in figure 1. Also, the type distribution of the never events is illustrated in figure 2 .

Urological Never Events in

a Tertiary-Level Center
Table 1. Numbers and percentages of the extra procedures indicated for the never events

\begin{tabular}{lclc}
\hline $\begin{array}{l}\text { Number of extra } \\
\text { procedures }\end{array}$ & $\begin{array}{l}\text { Patients } \\
\mathrm{n}(\%)\end{array}$ & \multicolumn{2}{c}{$\begin{array}{l}\text { Procedure per targeted } \\
\text { pathology event, n (\%) }\end{array}$} \\
\cline { 3 - 4 } & & Primary pathology & Never event \\
\hline Total extra procedures & $34(56)$ & $7(21)$ & $27(79)$ \\
One procedure & $18(30)$ & $6(33)$ & $12(67)$ \\
Two procedures & $13(21)$ & $1(8)$ & $12(92)$ \\
Three procedures & $3(5)$ & $0(0)$ & $3(100)$ \\
\hline
\end{tabular}

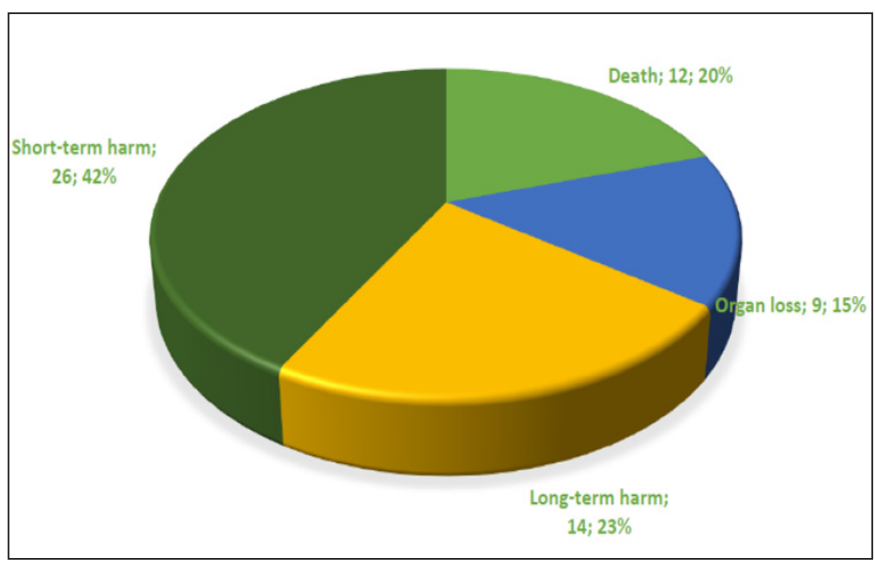

Fig. 3. Distribution of the final outcomes of the wrong-surgery never events.

In 1 case, the never event had a double occurrence in the primary and corrective extra-surgery, but with different operators.

The primary surgical procedure classes were major, intermediate, or minor in $65.5,11.5$, and $23 \%$ patients, respectively. Intraoperative discovery of the never event occurred in $35(57 \%)$ cases, while the others were discovered postoperatively. The number of extra-procedures and their targets after occurrence of the never events are described in table 1 . Twenty cases had one or more major extra-procedures.

A mistake from a qualified surgeon without any apparent underlying possible predisposing cause was found in $32(52 \%)$ events, while a disproportion between the procedure class and the urologist qualification level was found in $25(41 \%)$ events, and the emergence of an underlying undiagnosed anatomical anomaly in 4 (7\%) events. There were only 2 surgeons involved in more than 1 never event.

The final outcomes of all the described events are illustrated in figure 3 . Most of the 12 deaths occurred dur- 
ing or after major primary surgeries. Only 3 of them occurred after endoscopic procedures. Four deaths resulted from general urological procedures.

Among the 61 cases, only 20 (33\%) had announced official legal disputes with the primary operator or the institute in which the never event occurred.

\section{Discussion}

Unfavorable outcomes of urological surgical interventions may evolve from 3 possible scenarios. First, the primary surgery targets an extra-urological system and the surgeon is a non-urologist like a gynecological surgery-associated complication [3]. Second, the primary surgery targets a urological organ and was carried out by a urologist like during ureteroscopy [10]. Third, the primary surgery targets a urological organ, but the surgeon is a non-urologist, such as a circumcision done by a nurse [11]. Any of the 3 scenarios may result in human error-based harm which should not have occurred and, so, they are named surgical never events [6].

The real prevalence of the surgical never events appearrs to be more common than reported due to reluctance of reporting and the culture of blame, and involves all the surgical specialties $[9,12]$. Urological never events are more prevalent than other branches of surgery and represent up to $20 \%$ of all the never events [13]. This could be attributed to the anatomical nature of urinary and genital systems as being organ-paired [14]. Accordingly, wrong site events are more common in urology and could be attributed mainly to miscommunications [12].

In the current study and in spite of well-trained urologists, it was found that urological subspecialties which were involved in the management of urolithiasis (endourological, general urological, and open operations for urolithiasis) had the higher numbers of never events. This finding could be attributed to the high prevalence of the urolithiasis in Egypt, which indicated wide-range interventions from the non-invasive to the classic open surgery $[15,16]$. Also, simple open nephrectomy and transvesical prostatectomy techniques are subsequently still having their indications in our center under general urology.

In the current study, $20 \%$ of never events were referred from places outside our center for further managements. This percentage did not represent the actual incidence in the referred cases, because our center was not notified of all the events that occurred there. Also, they should not be put in the context of our events, because they may, incorrectly, appear higher.
The underlying contributing factors for surgical never events are variable and complex. Trials of reduction were targeted in many studies, but the situation is still demanding $[4,13]$. The aim is to decrease the surgical errors and improve patient safety through many targeted factors for quality and intervention in surgical system improvement [8]. The aim of the current study may cope with this aim where it exposes the prevailing situation in a tertiary-level urology center for proposed preventive purposes. It could be the first step for implementation of preventive organizational strategies in the context of the limited resources and unsatisfactory national evaluation systems.

Wrong side, wrong site, wrong patient, wrong procedure, wrong implant, retained foreign bodies, and missing surgical items are all included in the never events term [9]. The first 4 types are also included under the term wrong-site surgery with other minimally invasive interventional procedures and special procedures which expose the patient to more than minimal risk [1, 17]. Some authors tried to add complications such as pulmonary embolism, anesthesia-related complications, and infections to this term [5]. In the current study, these events were excluded because they are sequels or complications of possible occurrences due to other factors rather than the error factor and they are beyond the reach of the urologist's capabilities. Also, if they are included, this may make the definition of the never events redundant and it may lose its preventability as an important inclusion criterion [6].

Never events are classified into wrong-site surgery, retained surgical items, and surgical fires. Current prevalent estimates for the first 2 categories are 1 event per 100,000 and 1 event per 10,000 procedures, respectively [18]. The rate in the current results is similar. However, it may be preferable, in another way, to distinguish between the 3 groups among these events. First, the forgetting events such as retained foreign bodies or surgical items which are the most frequently seen, may need separate study. Second, the confusion events between 2 or more options such as wrong-side surgery in paired organs. Third, the technical error events such as wrong surgical procedure due to ignorance or negligence. For prevention, the former 2 groups need tools for reminding such as a surgical safety checklist which is very effective in minimizing the wrong-site surgery [7, 12]. The third group, however, needs tools for training and qualification of urologists [4].

In the results of the current study, wrong-site surgery represented only $7 \%$ and the majority were wrong procedure techniques. This could be explained by the major 
possible underlying causes of the individual urologist's inevitable mistakes and procedure class-surgeon level disproportion. The latter factor may indicate more effort for application of the procedure class-surgeon qualification level proportion policy. Although this policy is already employed, it appears to need more adherence to prevent possible unintended violations, especially during training of junior urologists. Also, this adherence should be considered with each up-grading of surgical procedure class-surgeon levels. This suggestion is based on the result that most of the surgical never events, in the current study, were in the major surgical procedure class.

Watson [19] discussed an important issue in never events when he referred to the responsibility shared by nurses along side doctors. This issue should be strongly advocated, in the training of nurses and awareness may help reduce non-technical error events. In the current study, 6 never events were related to nursing defects in perioperative preparations.

Regarding the current results, one may be surprised about the final outcomes which included deaths and permanent organ losses. These outcomes cause stress to the patients and their families because of the physical or psychological losses from just skin wounding up to loss of life. Also, they impact the performance of the urologists and their teams who may blame themselves for these errors and may take a long time to recover [14].

Accordingly, urological never events are costly in 2 ways. The first is that they exhaust the mentalities of the patients, the urologists, and administrative authorities. The other cost is a financial one where the extra expenses for the extra procedures and legal compensations are considerable [7]. In the current results, only $20 \%$ of the cases had officially announced legal disputes. This low percentage could be attributed to resolving of the disputes through traditional ways such as unannounced financial and social compensations for the patient about the shortterm harms or sometimes just blaming of or forgiving the operator. However, discussion of the administrative steps from the hospital leadership towards certain surgeons or nurses who had documented unreasoned involvement in the occurrence of these never events were beyond the scope of this article.

In this article, the urological surgical never events were described by the term "catastrophe" to expose their unfavorable effects. However, it should not be confused with the term "catastrophe surgery", because the latter was coined for the surgical responses to multiple causalities and devastating injuries which result from environmental catastrophes [20].
The results of the current study only reflect the situation in a single tertiary-level urology center in Egypt. So, urological surgical never events may need national and global study projects to determine the situations in the other centers and the most responsible factors. Large-volume centers should expose their experiences without reluctance to revise the causes and plan for improved system correction by training, surgeon subspecialty- and level-restricted surgery policies, and new technology utilization for operative adjustments to decrease inevitable human errors as far as possible. The current study may motivate other national centers and hospitals to design larger studies to consider the never events problem.

\section{Conclusion}

Surgical never events may involve all the urological subspecialties and may result in disastrous outcomes up to patient death. Although they are rare events and potentially preventable, practically this issue is still a difficult and demanding realty due to the complex underlying factors including inevitable human errors, and unpredictable anatomical anomalies. However, reduction to the minimum is a mandatory target that should be adopted in all healthcare providing facilities by training of clinicians, surgeon subspecialty- and level-restricted surgery policies, and strict administrative policies. Large-volume and tertiary-level centers should lead the projects of prevention through both the medical and nursing fields. 


\section{References}

1 Comprehensive Accreditation Manual for Hospitals (CAMH) Update; Sentinel events. 2016 Jan. Available at: http://www.jointcommission.org. Accessed 11 Jan. 2017, p20.

2 Dindo D, Demartines N, Clavien PA: Classification of surgical complications: a new proposal with evaluation in a cohort of 6336 patients and results of a survey. Ann Surg 2004;240:205-213.

3 Eke N: Urological complications of abdominal surgery. J West Afr Coll Surg 2013;3:912.

4 Algie CM, MR, Wasiak J, Batty L, Gruen RL, Mahar PD: Interventions for reducing wrong-site surgery and invasive clinical procedures. Cochrane Database Syst Rev 2015: CD009404.

5 Makar A, Kodera A, Bhayani SB: Never events in surgery. Eur Urol 2015;68:919-920.

6 National Quality Forum (NQF): Serious reportable events in healthcare-2011 update: A consensus report. Available at: www.qualityforum.org. Accessed 2017 Jan 4, Washington, DC, USA.

7 Haynes AB, Weiser TG, Berry WR, Lipsitz SR, Breizat AH, Dellinger EP, Herbosa T, Joseph S, Kibatala PL, Lapitan MC, Merry AF, Moorthy K, Reznick RK, Taylor B, Gawande AA: A surgical safety checklist to reduce morbidity and mortality in a global population. N Engl J Med 2009;360:491-499.
8 Thiels CA, Lal TM, Nienow JM, Pasupathy KS, Blocker RC, Aho JM, Morgenthaler TI, Cima RR, Hallbeck S, Bingener J: Surgical never events and contributing human factors. Surgery 2015;158:515-521.

9 Seiden S, Barach P: Wrong-side/wrong-site, wrong-procedure, and wrong-patient adverse events: are they preventable? Arch Surg 2006;141:931-939.

10 Watterson J, Mahoney JE, Futter NG, Gaffield J: Iatrogenic ureteric injuries: approaches to etiology and management. Can J Surg 1998;41:379-382.

11 Eke N: Iatrogenic urological trauma: a 10year experience from Port Harcourt. West Afr J Med 2000;19:246-249.

12 Hanchanale V, Rao AR, Motiwala H, Karim OM: Wrong site surgery! How can we stop it? Urol Ann 2014;6:57-62.

13 Alleemudder A, King P, Mehta S: Point of technique: reducing wrong-side errors for endourology procedures. Urology 2014;84: 1541-1543.

14 Challacombe B, Dasgupta P, Amoroso P, Kirby R: Wrong-side/site surgery. Trend Urol Men Health 2011;2:32-34.

15 Elderwy A, Kurkar A, Hussein A, Abozeid H, Hammodda HM, Ibraheim AF: Dissolution therapy versus shock wave lithotripsy for radiolucent renal stones in children: a prospective study. J Urol 2014;191(5 Suppl): 1491-1495.
16 Al-Kohlany K, Shokeir AA, Mosbah A, Mohsen T, Shoma AM, Eraky I, El-Kenawy M, El-Kappany HA: Treatment of complete staghorn stones: a prospective randomized comparison of open surgery versus percutaneous nephrolithotomy. J Urol 2005;173: 469-473.

17 Carayon P, Schultz K, Hundt AS: Righting wrong site surgery. Jt Comm J Qual Saf 2004;30:405-410.

18 Hempel S, Maggard-Gibbons M, Nguyen DK, Dawes AJ, Miake-Lye I, Beroes JM, Booth MJ, Miles JN, Shanman R, Shekelle PG: Wrong-site surgery, retained surgical items, and surgical fires: a systematic review of surgical never nvents. JAMA Surg 2015; 150:796-805.

19 Watson DS: Concept analysis: wrong-site surgery. AORN J 2015;101:650-656.

20 McAlister VC: Catastrophe surgery: response to multiple casualties or individual patients with devastating injuries. Can J Surg 2009; $52: 175-176$ 